\title{
Alignment of Rod-Shaped Gold Particles by Electric Fields
}

\author{
Bianca M. I. van der Zande, ${ }^{* \dagger, \ddagger}$ Ger J. M. Koper, ${ }^{\S}$ and Henk N. W. Lekkerkerker*, ${ }^{\dagger}$ \\ Van't Hoff Laboratory for Physical and Colloid Chemistry, Debye Institute, Utrecht University, Padualaan 8, \\ 3584 CH Utrecht, The Netherlands, Leiden Institute of Chemistry, Leiden University, Gorlaeus Laboratories, \\ P.O. Box 9502, 2300 RA Leiden, The Netherlands, and Philips Research Laboratories, Prof. Holstlaan 4, \\ 5656 AA Eindhoven, The Netherlands
}

Received: December 14, 1998; In Final Form: April 15, 1999

\begin{abstract}
The electro-optical response of colloidal dispersions of rod-shaped gold particles is studied for various aspect ratios $(2.6<L / d<49 ; d=15 \mathrm{~nm})$ by monitoring the absorbance spectra in the visible wavelength regime. The absorbance spectra strongly depend on the degree of orientational order of the rods and the direction of the polarization of the incident light with respect to the applied electric field. From the change in absorbance of the transverse resonance the electro-optical effect is deduced: at low electric field strengths a linear dependence on the squared electric field strength is observed, and at sufficiently high electric field strengths saturation occurs. This is in agreement with electro-optical theory. With increasing length, the anisotropy in electric polarizability increases from $6 \cdot 10^{-30} \mathrm{~F} \cdot \mathrm{m}^{2}$ to $12 \cdot 10^{-30} \mathrm{~F} \cdot \mathrm{m}^{2}$. It is found that the anisotropy in electric polarizability depends on the square root of the particle length.
\end{abstract}

\section{Introduction}

The electrical alignment of anisotropic particles such as tobacco mosaic virus (TMV), ${ }^{1,2} \mathrm{FeOOH},{ }^{3-5}$ and attapulgite ${ }^{6}$ is often studied by electro-optical techniques ${ }^{7-9}$ (i.e. electric birefringence, electric light scattering, and dichroism). These techniques are useful tools to monitor the geometrical ${ }^{4,5,10}$ and electrical properties ${ }^{5,11}$ of dispersed anisotropic particles. Apart from characterization of the particle properties, electro-optical phenomena are of fundamental interest because they give insight into alignment mechanisms, ${ }^{3,5,12-14}$ double layer properties, ${ }^{3,15,16,17}$ and long-range interactions. ${ }^{13,14,18}$

In this paper the alignment of rod-shaped gold particles is studied by measuring the absorbance spectra of the rod dispersions as a function of the electric field strength. In the absence of an electric field the gold rods are randomly oriented. Consequently, the absorbance spectra display two maxima: the transverse and the longitudinal resonance. In the presence of an electric field the relative magnitude of the resonances is sensitive to the induced orientational order in the rod dispersion (Figure 1). When the incident light is polarized parallel to the applied electric field, disappearance of the transverse resonance can be achieved by fully aligning the gold particles, while the longitudinal resonance remains present. For perpendicularly polarized light the transverse resonance survives at the expense of the longitudinal resonance. In ref 19 we report on a study of these phenomena as a result of alignment of the particles by the stretched film method. ${ }^{20,21}$ In such films complete alignment is achieved because of the absence of Brownian motion. In aqueous dispersions complete alignment is only obtained at experimentally unattainable high electric field strengths.

$\dagger$ Utrecht University.

$\doteqdot$ Philips Research Laboratories.

$\S$ Leiden University.

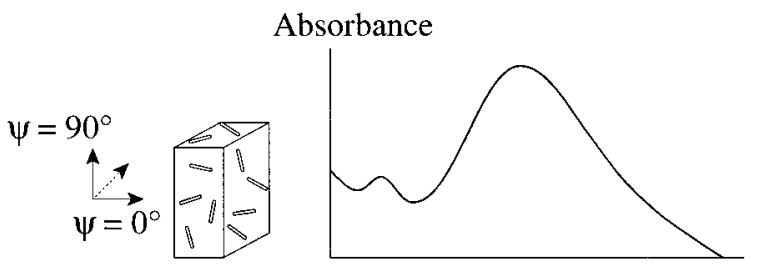

a)
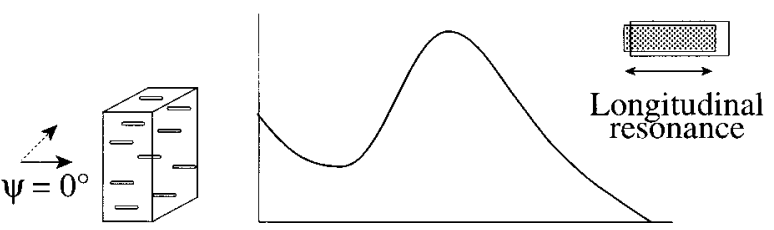

b)

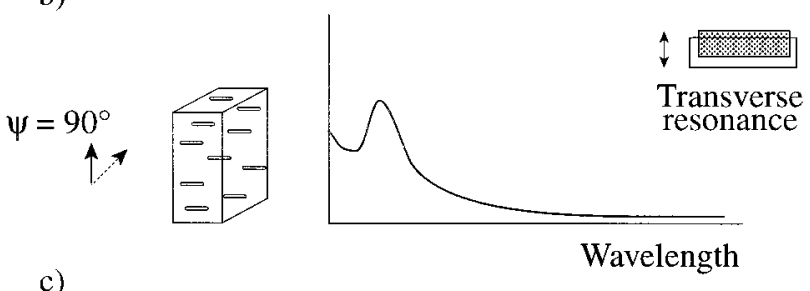

c)

Figure 1. Schematic representation of the absorbance of light by colloidal dispersions of rod-shaped gold particles. (a) The absorbance spectrum for randomly oriented gold rods displays both the longitudinal and the transverse resonance for unpolarized, parallel polarized, and perpendicular polarized light. The absorbance spectrum for aligned gold rods displays (b) only the longitudinal resonance in the case of parallel polarized light and (c) only the transverse resonance in the case of perpendicular polarized light. The schematics in the absorbance spectra indicate the direction of the plasmon oscillation with respect to the main axis of the particle and the corresponding resonance. The dotted arrow indicates the wavevector of the incident light. 


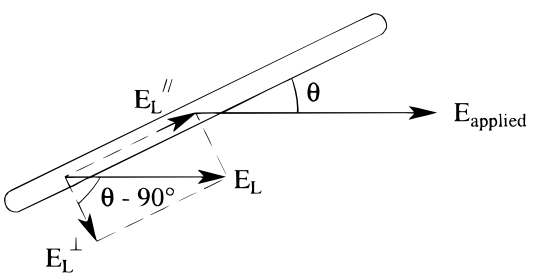

Figure 2. Orientation of the particle with respect to the applied electric field $(E) . \theta$ is the angle of the long axis of the particle with the applied electric field. The components of the electric field of the incident light $\left(E_{\mathrm{L}}\right)$ perpendicular $\left(E_{\mathrm{L}}^{\perp}\right)$ and parallel $\left(E_{\mathrm{L}}{ }^{\prime \prime}\right)$ to the main axis are also indicated.

For gold rods of $15 \mathrm{~nm}$ diameter, the transverse resonance occurs in the visible wavelength regime around $520 \mathrm{~nm}$. The longitudinal resonance depends on the aspect ratio. For rods with $L / d=2.6$, the longitudinal resonance occurs around 720 $\mathrm{nm}$, while for $L / d=12$ the band is shifted $1000 \mathrm{~nm}$ to the near-infrared region and appears around $1700 \mathrm{~nm} .{ }^{22}$ Since in this study the longitudinal resonance lies beyond the experimental wavelength regime, only the behavior of the transverse resonance is investigated as a function of the electric field strength. The results are interpreted by electro-optical theory as described in, for example, refs 1 and 7-9. Subsequently, the anisotropy in electric polarizability of gold rods with various aspect ratios is deduced from the electro-optical behavior of the transverse resonance. For comparison, some results from electric birefringence measurements are presented as well. ${ }^{23}$

This paper is organized as follows. Section 2 presents the electro-optical equations originally formulated by Peterlin and Stuart. ${ }^{24}$ Analytical expressions for the angular distribution were derived by $\mathrm{O}^{\prime}$ Konski. ${ }^{1,7,9}$ In section 3 the experimental details are presented. We discuss the results in section 4 .

\section{Theory}

The optical properties of a dispersion of rod-shaped particles change under the influence of an external electric field due to the alignment of the particles. The relative difference in magnitude of the optical property measured in the presence of an electric field, $B_{\mathrm{E}}$, and without electric field, $B_{0}$, is called the electro-optical response ${ }^{1,7}$

$$
G_{\mathrm{E}}=\frac{B_{\mathrm{E}}-B_{0}}{B_{0}}
$$

This holds for various optical effects such as birefringence or dichroism.

The steady state electro-optical response $e^{1,7,9}$ can be expressed as the product of two functions: the orientation factor, $F$, and the saturation value at infinitely high electric field strengths, $G_{\infty}$.

$$
G_{\mathrm{E}}=G_{\infty} F(E)
$$

with

$$
F(E)=\frac{3}{2}\left\langle\cos ^{2} \theta\right\rangle_{E}-\frac{1}{2}
$$

Here, $\theta$ represents the angle between the long axis of the particle and the direction of the applied electric field, as illustrated in Figure 2. $F$ is the orientation factor obtained as the average over the angular distribution of the particles in the electric field $E$. The orientation factor depends on the electric field strength $E$, thermal energy $k T$, and the anisotropy in electric polarizability

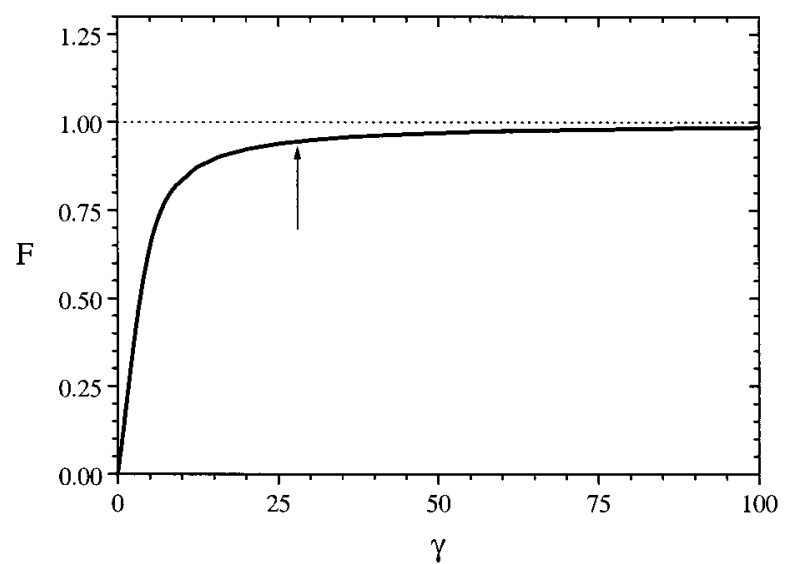

Figure 3. The orientation factor $F$ plotted against $\gamma\left(=\Delta \alpha E^{2} / k T\right){ }^{1}$ The maximum $\gamma$ in the experiments is indicated by the arrow.

$\Delta \alpha$ of the particles to be discussed later. The saturation value of the electro-optical effect for fully aligned particles, $G_{\infty}$, depends on particle shape and size and optical properties as well as on the geometry of the experimental set up. ${ }^{7}$

The average orientation of the rods with respect to the applied electric field is a result of the competition between the energy of orientation and the Brownian energy of the particles. In the case of induced dipoles, the potential energy of orientation is

$$
U=-\frac{1}{2} \Delta \alpha E^{2} \cos ^{2} \theta
$$

with $\Delta \alpha=\alpha_{\|}-\alpha_{\perp}$, the anisotropy in electric polarizability, where the electric polarizabilities parallel and perpendicular to the main axis of the particle are denoted by $\alpha_{\|}-\alpha_{\perp}$, respectively. Subsequently, an analytical expression for the angular distribution of noninteracting particles can be obtained by using a Boltzmann factor of the orientation energy (eq 3). O'Konski et al. ${ }^{1}$ derived the expression for the case of electric birefringence. This expression can also be used for electric light scattering and dichroism. ${ }^{7}$ For induced dipole moment orientation it reduces to

$$
F=\frac{3}{4}\left[\frac{\mathrm{e}^{\gamma}}{\sqrt{\gamma} \mathrm{D}(\gamma)}-\frac{1}{\gamma}\right]-\frac{1}{2}
$$

with

$$
\gamma=\frac{\Delta \alpha E^{2}}{2 k T} \text { and } D(\gamma)=\int_{0}^{\gamma} \mathrm{e}^{\mathrm{t}^{2}} \mathrm{~d} t
$$

In Figure 3 the orientation factor is plotted versus the scaled electric field strength $\gamma$. At extremely high electric field strengths $(\gamma \rightarrow \infty)$, the particles are fully aligned and $F$ becomes unity: the electro-optical response saturates. For low degrees of orientation eq 4 simplifies to ${ }^{7,9}$

$$
F=\frac{\Delta \alpha E^{2}}{15 k T}
$$

Consequently, $F$ and $G / G_{\infty}$ (see eq 2 ) are linear functions of the electric field strength squared with a slope equal to $\Delta \alpha / 15 k T$ in the low orientational order regime $\left(|\Delta \alpha| E^{2} \ll k T\right)$.

In the present case the electro-optical effect is deduced from the behavior of the transverse resonance in external electric fields. The transverse resonance is probed by the electric field component of the incident light perpendicular to the main axis of the particle (see Figure 1). This component, as illustrated in 
Figure 2, is equal to

$$
E_{\mathrm{L}}^{\perp}=E_{\mathrm{L}} \cos \left(\frac{\pi}{2}-\theta\right)=E_{\mathrm{L}} \sin \theta
$$

where $E_{\mathrm{L}}$ is the electric field strength of the incident light and $\theta$ the angle between the main axis of the rod and the applied electric field. The absorbance is proportional to the average angular distribution of the perpendicular component of the electric field strength squared of the incident light $\left\langle\left(E_{\mathrm{L}}^{\perp}\right)^{2}\right\rangle$. Consequently, the magnitude of the transverse resonance is sensitive to the particle orientation with respect to the electric field of the incident light. For instance, if particles are fully aligned, $\theta=0$, and hence $\left\langle\left(E_{\mathrm{L}}{ }^{\perp}\right)^{2}\right\rangle=0$, which results in an absence of the transverse resonance, whereas in the random situation $\left\langle\left(E_{\mathrm{L}}^{\perp}\right)^{2}\right\rangle=2 / 3 E_{\mathrm{L}}^{2}$.

In this study the electro-optical effect is calculated as follows

$$
\frac{G}{G_{\infty}}=\frac{A_{E}-A_{0}}{A_{E_{\mathrm{s}}}-A_{0}}
$$

Here $A_{E}$ is the absorbance measured at an applied electric field strength $E$ with light polarized parallel to the applied electric field, $A_{E_{\mathrm{s}}}$ is the saturation absorbance at $E \geq E_{\mathrm{s}}$ with $E_{\mathrm{s}}$ the saturation electric field strength, and $A_{0}$ is the absorbance without an applied electric field. Taking into account that the change in the absorbance is proportional to $\left(E_{\mathrm{L}}^{\perp}\right)^{2}$ and that averaging over all possible angles has to be performed, the combination of eqs 6 and 7 results in

$$
F=\frac{G}{G_{\infty}}=\frac{\left\langle\sin ^{2} \theta\right\rangle-\frac{2}{3}}{0-\frac{2}{3}}=\frac{3}{2}\left\langle\cos ^{2} \theta\right\rangle-\frac{1}{2}
$$

which is exactly the orientation factor used in eq 2 . Since the behavior of the transverse resonance depends on the same orientation factor as the birefringence, we will use eq 4 to relate the eletro-optical behavior of the transverse resonance to an orientational ordering. Subsequently, the anisotropy in electric polarizability is deduced from the measured orientation factor, $F$, provided that the alignment is only due to induced dipoles.

\section{Experimental Section}

Aqueous Dispersions of Rod-Shaped Gold Particles. Five dispersions of rod-shaped gold particles with various aspect ratios were synthesized and characterized as described in refs 25 and 26, respectively. The synthesis is based on the electrodeposition of gold in the nanopores of alumina, followed by dissolution of the membrane and release of the rods from the electrode. During the dissolution of the membrane poly(vinylpyrrolidone) (K30-PVP, $M_{\mathrm{w}}=40000$, Fluka) was added to stabilize the gold rods. The thickness of the PVP layer is estimated to be $10-15 \mathrm{~nm}$. Apart from stabilization based on steric repulsion, stabilization also results from double layer repulsion because the rods are negatively charged with an average $\zeta$ potential of $-47 \pm 7 \mathrm{mV}$. The rod diameter is determined by the pore diameter, which equaled $15 \pm 3 \mathrm{~nm}$, and the rod length, $L$, was tuned in the range $39 \mathrm{~nm}<L<730$ $\mathrm{nm}$ by varying the gold deposition time. The particle dimensions were obtained from transmission electron microscopy (TEM). About 30-100 particles were analyzed. The number-averaged lengths and diameters as well as their standard deviations are presented in Table 1. The systems are coded as, for example, ROD12.6, in which the number refers to the aspect ratio.
TABLE 1: Characteristics of the Template-Synthesized Gold Rods Dispersed in an Aqueous Medium ${ }^{25}$ a

\begin{tabular}{lccccccc}
\hline & $L / d$ & $\begin{array}{c}L \\
(\mathrm{~nm})\end{array}$ & $\begin{array}{c}\sigma_{L} \\
(\mathrm{~nm})\end{array}$ & $\begin{array}{c}d \\
(\mathrm{~nm})\end{array}$ & $\begin{array}{c}\sigma_{d} \\
(\mathrm{~nm})\end{array}$ & $\begin{array}{c}c_{\mathrm{Au}} \\
(\mu \mathrm{g} / \mathrm{mL})\end{array}$ & $\begin{array}{c}c_{\text {rods }} \\
\left(10^{10} \mathrm{~mL}^{-1}\right)\end{array}$ \\
\hline SPHERE & & & & 15 & 3 & 46 & \\
ROD2.6 & 2.6 & 39 & 10 & 15 & 3 & 30 & 20 \\
ROD12.6 & 12.6 & 189 & 24 & 15 & 3 & 131 & 20 \\
ROD17.4 & 17.4 & 279 & 68 & 16 & 3 & 28 & 2.9 \\
ROD49 & 49 & 729 & 20 & 15 & 3 & 145 & 2.8 \\
ROD17.2 & 17.2 & 259 & 60 & 15 & 2 & 24 & 2.7
\end{tabular}

${ }^{a}$ The particle dimensions are determined by TEM analysis and the total gold concentration $\left(c_{\mathrm{Au}}\right)$ is determined by ICP optical emission spectroscopy. The bottom line refers to the experimental system used in ref 23. $L=$ the number-averaged particle length. $d=$ the numberaveraged diameter. $\sigma_{x}=$ the standard deviation of $x . c_{\text {rods }}=$ the number concentration of rods calculated with eq 11. Note: the maximum relative inaccuracy in the total gold concentration is $3 \%$, whereas the accuracy of the number concentration of rods strongly depends on the accuracy of the average particle length and diameter.

Table 1 includes the total gold concentration $\left(g \cdot \mathrm{L}^{-1}\right)$ and the number concentration of rods. The number concentration of rods $\left(c_{\text {rods }}\right)$ was estimated as follows

$$
c_{\text {rods }}=\frac{c_{\mathrm{Au}}}{\rho_{\mathrm{Au}} \pi r^{2} L}
$$

Here $L$ is the average particle length, $r$ the average radius both determined by analysis of transmission electron micrographs, $c_{\text {Au }}$ the total gold concentration determined with inductively coupled plasma-emission spectroscopy (maximum relative inaccuracy of $3 \%)$, and $\rho_{\text {Au }}$ represents the mass density of gold ( $\rho_{\text {Au }}$ $\left.=19.310^{3} \mathrm{~kg} \cdot \mathrm{m}^{-327} \mathrm{j}\right)$. Spherical gold particles with $d=15$ $\mathrm{nm}$ were prepared following the method of Frens. ${ }^{28}$ PVP (4 $\mathrm{g} \cdot \mathrm{L}^{-1}$ ) was added to obtain PVP-stabilized spheres. This spherical gold sol served as a reference. Table 1 includes the characteristics of the spherical gold sol.

Methods. Absorbance Measurements. The set up and electrooptical cell are shown in Figure 4. The electro-optical cell consisted of two stainless steel electrodes $(3 \times 1 \times 0.35 \mathrm{~cm})$ (1) coated with platinum, which were placed in a cuvette with optical path length $x=1 \mathrm{~cm}$. The roughness $\left(R_{\mathrm{a}}\right)$ of the electrodes was $0.276 \mu \mathrm{m}$ determined by atomic force microscopy. The sample volume was approximately $0.5 \mathrm{~mL}$. The electrodes were separated $1 \mathrm{~mm}$ by two Teflon spacers (2). The ac voltage was applied for $1 \mathrm{~s}$ with a function generator (Philips PM 5136 function generator, $0.1 \mathrm{mHz}$ to $5 \mathrm{MHz}$ ) coupled to an amplifier (TOE 7608 wide-band amplifier TOELLNER), with amplification factor 7 . The electrodes were connected with the amplifier via a cap (3) which ensures a reliable contact between the electrodes and the amplifier. The measurements were performed as a function of the electric field strength ranging from 0 to $140 \mathrm{~V} \cdot \mathrm{mm}^{-1}$ at a frequency of $10 \mathrm{kHz}$. The voltage is increased in steps of $7 \mathrm{~V}$. After each measurement at a particular voltage, the voltage was switched off for about $10 \mathrm{~s}$, before applying the next, allowing randomization of the particle orientation by Brownian motion.

The absorbance spectra $(\gamma=400-800 \mathrm{~nm})$ were measured during $1 \mathrm{~s}$ on a diode array spectrophotometer (Hewlett Packard 8452 A) using water as a reference. The optical set up is shown in Figure 4. A VIS polarizer (Spindler \& Hoyer) was mounted in front of the cuvette. The spectra were measured at a polarization angle $\Psi=0^{\circ}$ and $\Psi=90^{\circ}$.

Subjecting dispersions to electric fields may lead to a considerable Joule heating. ${ }^{8}$ In this study Joule heating incidently occurred at field strengths exceeding $100 \mathrm{~V} \cdot \mathrm{mm}^{-1}$, which leads 


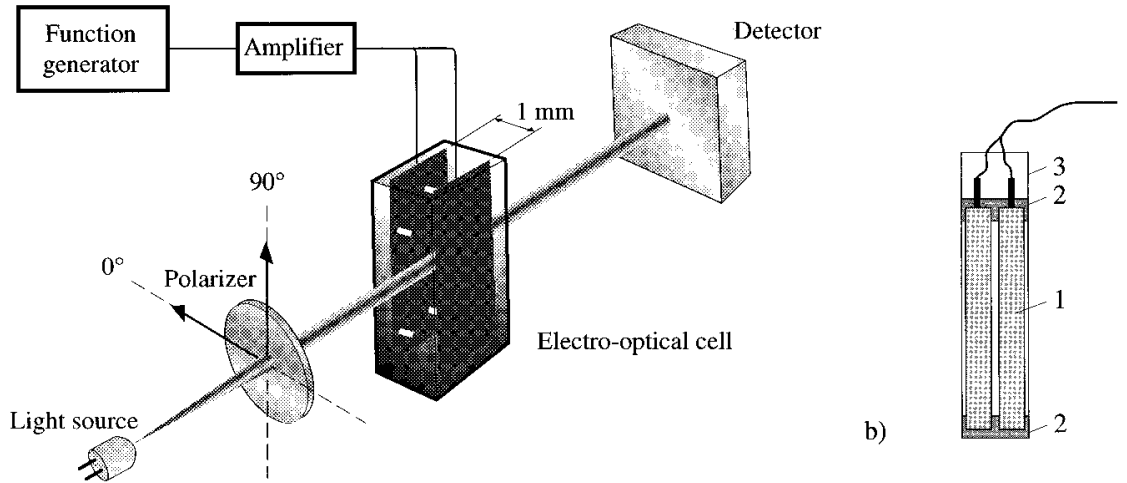

a)

Figure 4. (a) Optical set up. In front of the light source a polarizer is mounted. At $\Psi=0^{\circ}$ the applied electric field is parallel to the polarization direction of the incident light and at $\Psi=90^{\circ}$ the polarization direction is perpendicular to the applied electric field. The electric field is applied by a function generator coupled with an amplifier. (b) The electrooptical cell. In an ordinary cuvette, two electrodes (1) are placed at a distance of $1 \mathrm{~mm}$ realized by two spacers (2), one on the bottom and one on the top. The connection with the amplifier is achieved by a specially designed cap (3) which ensures a reliable contact between the electrodes and the amplifier.
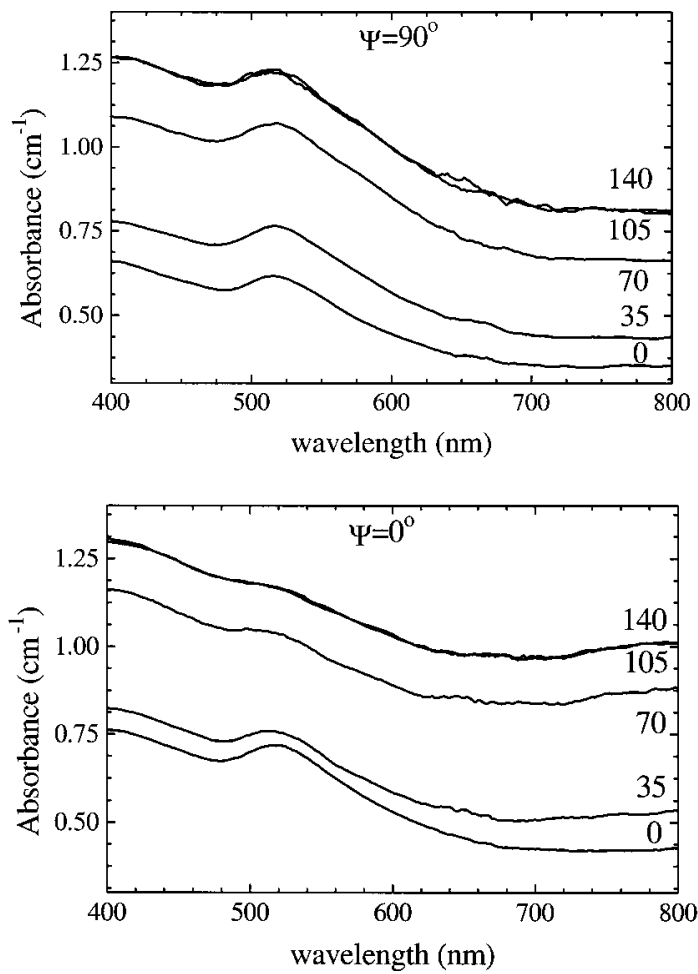

Figure 5. The absorbance spectra obtained for ROD49. The numbers on the spectral curves represent the electric field strength $\left(\mathrm{V} \cdot \mathrm{mm}^{-1}\right)$

to temperature gradients. In this regime the sample was cooled down after one measurement. Accordingly, Joule heating did not affect the presented measurements significantly.

Effect of Duration of the Electric Field. The effect of duration of the electric field was examined visually for a PVP-stabilized gold sol with $L / d=39$ on a time scale of minutes. The same electro-optical cell was used. The ac voltage was applied for several minutes with the function generator coupled to a homebuilt amplifier with amplification factor 50. The measurements were performed at an ac voltage of 25,125 , or $175 \mathrm{~V}$, with a frequency of $100 \mathrm{kHz}$.

\section{Results and Discussion}

The Behavior of the Transverse Resonance in an Electric Field. Figures 5, 6, and 7 respectively show the spectral curves obtained for the dispersions ROD49, ROD12.6, and ROD2.6
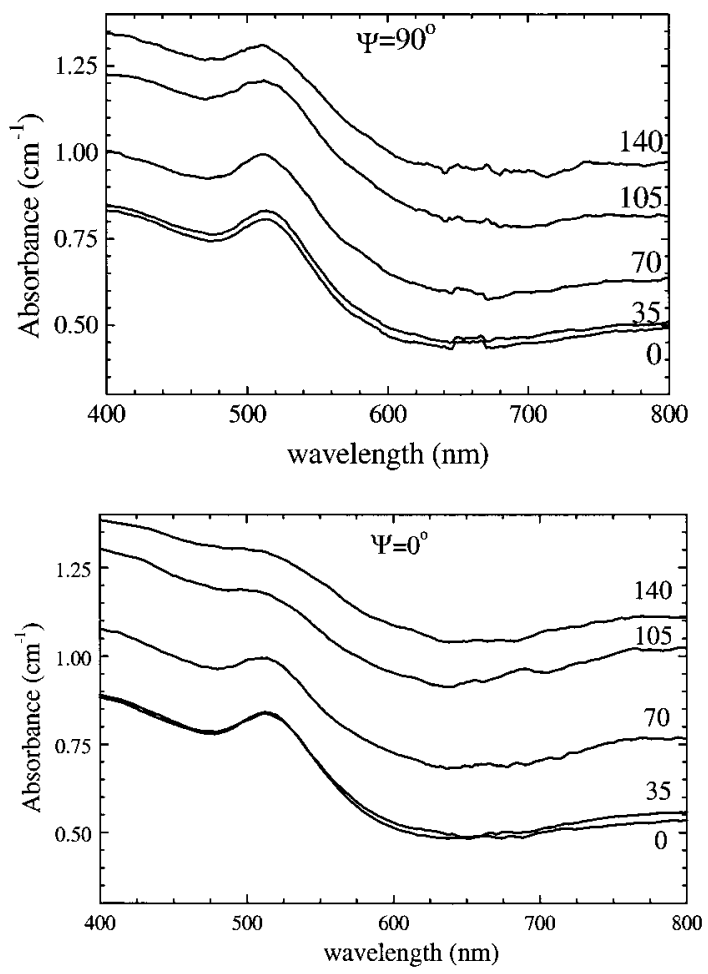

Figure 6. The absorbance spectra obtained for ROD12.6. The numbers on the spectral curves represent the electric field strength $\left(\mathrm{V} \cdot \mathrm{mm}^{-1}\right)$.

at $\Psi=0^{\circ}$ and $\Psi=90^{\circ}$. The absorbance behavior of dispersion ROD17.4 is similar to that of ROD49 and is therefore not shown. Two effects are present in the spectra for the rod dispersions by applying an electric field: disappearance of the transverse resonance at $\Psi=0^{\circ}$ and a shift of the spectral curves to higher absorbance values. This shift is reversible and can be interesting for applications such as smart windows. ${ }^{29,30}$ As discussed in the theory section, we focus on the disappearance of the transverse resonance. As a reference we have examined the electro-optical behavior of gold spheres. The absorbance for spherical gold sols is not affected by the polarization of light and the applied electric field, as is expected. This is a strong indication that the applied electric field does not induce aggregation on the time scale of $1 \mathrm{~s}$. Furthermore, the optical properties of gold do not significantly change upon external fields, nor is there a significant electrically induced anisotropy. Therefore, the presence of an electro-optical response in the 

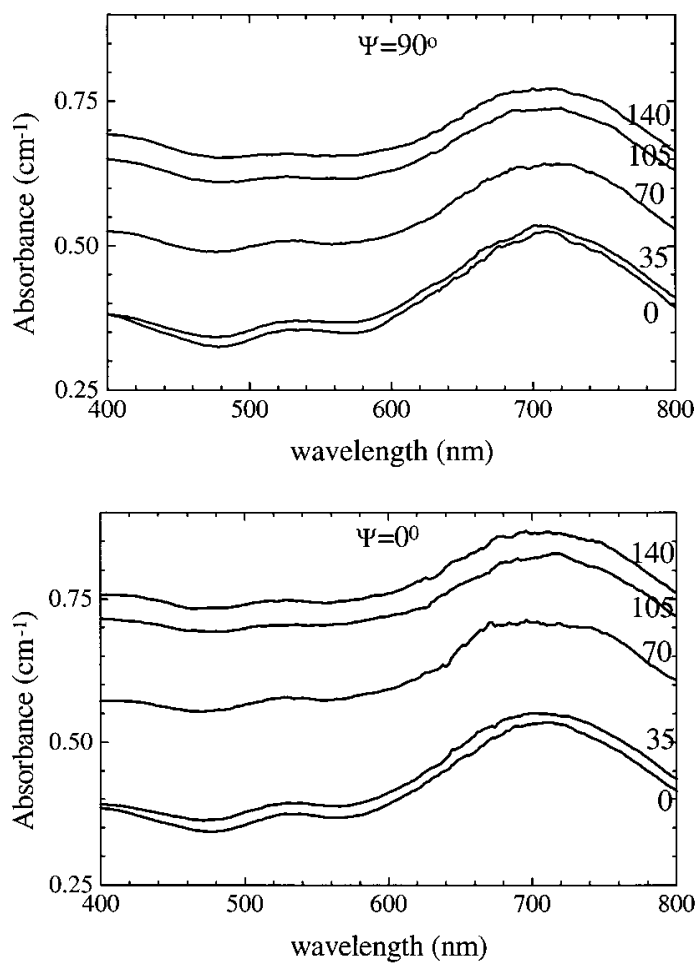

Figure 7. The absorbance spectra obtained for ROD2.6. The numbers on the spectral curves represent the electric field strength $\left(\mathrm{V} \cdot \mathrm{mm}^{-1}\right)$. Note that in this particular case both resonances are present: the transverse resonance occurs around $520 \mathrm{~nm}$, whereas the longitudinal resonance occurs around $750 \mathrm{~nm}$.

case of gold rods can be ascribed to their orientation in applied electric fields.

Focusing on the absorbance behavior of ROD 49 at $\Psi=0^{\circ}$ (Figure 5), the transverse resonance (around $520 \mathrm{~nm}$ ) gradually decreases with increasing electric field, whereas at $\Psi=90^{\circ}$ the transverse resonance seems to be unaffected. This indicates that the particles are oriented by the external electric field. Complete alignment is, however, not yet obtained, which is indicated by the very weak shoulder in Figure 5. Note that the longitudinal resonance will be in the infrared and thus cannot be monitored with the apparatus used. A similar behavior is found for the system ROD12.6 (Figure 6). However, at $E=$ $140 \mathrm{~V} \cdot \mathrm{mm}^{-1}$ and $\Psi=0^{\circ}$, the transverse resonance shows a small shoulder, illustrated in Figure 7, which is considerably larger than in the case of ROD17.4 and ROD49. Furthermore, the maximum electric field strength applied in this study is insufficient to monitor a significant change in the resonance bands for the system ROD2.6 as shown in Figure 7. Only a shift of the spectral curves to higher absorbance values is observed. Because the size distribution of the system ROD2.6 is much broader than for the other systems, the electrically induced optical behavior may not be that pronounced. This particular system consists of rod-shaped, ellipsoidal, and spherical particles. ${ }^{26}$ The presence of various particle shapes may affect the electro-optical response.

Steady-State Electro-Optical Effect. To obtain an electrooptical reponse we subtracted the spectral curve measured without field $(E=0)$ from the spectral curve measured with an applied field $(E \neq 0)$. Figure 8 shows an example. Instead of a disappearance of the transverse resonance, a reversed peak is obtained and the baseline becomes almost horizontal. Since we cannot apply sufficiently high electric field strengths, complete alignment $(F=1)$ is experimentally unattainable. Therefore, we will analyze our data in the pseudosaturation regime at which

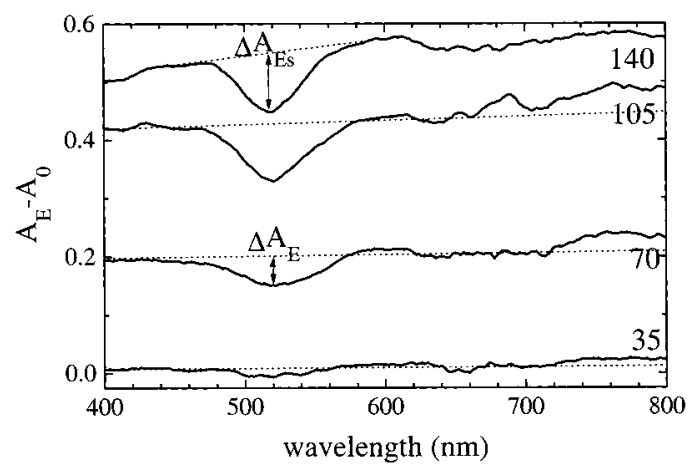

Figure 8. The spectral curves of ROD12.6 presented in Figure 6 are replotted by subtracting the spectral curve measured at $E=0$ from the curves measured in presence of an electric field. $\Delta A_{E}$ represents the well depth, which increases with increasing electric field, and $\Delta A_{E_{\mathrm{S}}}$ the saturation value for the well depth.

the experimental electro-optical effect (eq 1) appears to saturate. Note that by analyzing the data in the pseudosaturation regime, $F$ is overestimated by about $10 \%$ (see Figure 3 ). The normalized electro-optical effect is calculated as

$$
\frac{G}{G_{\max }}(\approx \mathrm{F})=\frac{\Delta A_{E}}{\Delta A_{E_{\mathrm{s}}}}
$$

where $G_{\max }$ is the experimental saturation value for $G$. Here $\Delta A_{E}$, indicated in Figure 8, represents the well depth at $E \neq 0$, and $\Delta A_{E}$ s the saturation value. We were not able to deduce a saturation electro-optical effect for ROD2.6 from the transverse or longitudinal resonance, because the orientational sensitivity of the plasmon resonances is insufficient.

Figure 9 represents the field strength dependence of the electro-optical effect of colloidal gold rods with aspect ratios $L / d=49,17.4$, and 12.6. As predicted by theory (Figure 3 ), at low electric fields the measured electro-optical response is linear with the squared applied electric field corresponding to low degrees of orientation. At higher electric fields, deviation of this linear behavior is observed until a steady-state value is reached. Using the $\Delta \alpha$, which can be estimated from the slope of the linear part of the curves shown in Figure 9 (by means of eqs 2 and 5), we are able to calculate $\gamma\left(=\Delta \alpha E^{2} / 2 k T\right)$. Table 2 summarizes the anisotropy in electric polarizability. Subsequently, the experimental electro-optical effect is replotted as a function of $\gamma$ in Figure 10. Figure 10 includes the theoretical curve for induced dipole orientation derived by O'Konski in the $\gamma$ range $0-30$. The experimental data are close to the calculations.

Figure 10 also demonstrates that the maximum value for $\gamma$ depends on the particle length. The length dependence of the maximum $\gamma$ is in agreement with the relative magnitudes of the transverse resonance band. With increasing rod length, the maximum $\gamma$ increases, and therefore higher degrees of alignment can be obtained. The smallest contribution at $E=140 \mathrm{~V} \cdot \mathrm{mm}^{-1}$ of the transverse resonance is indeed obtained by system ROD49 (Figure 5). With decreasing $\gamma$, the transverse resonance contribution at $E=140 \mathrm{~V} \cdot \mathrm{mm}^{-1}$ increases (ROD12.6, Figure 6) and at very small values of $\gamma(\gamma<7)$ the electro-optical effect does not saturate at all, which may partially explain the apparent insensitivity of the resonances to the polarization direction and electric field strength in the case of the dispersion ROD2.6 (Figure 7).

Anisotropy in Electric Polarizability and the Saturation Voltage. The anisotropy in electric polarizability (calculated with eqs 2 and 5) increases with increasing particle length from 

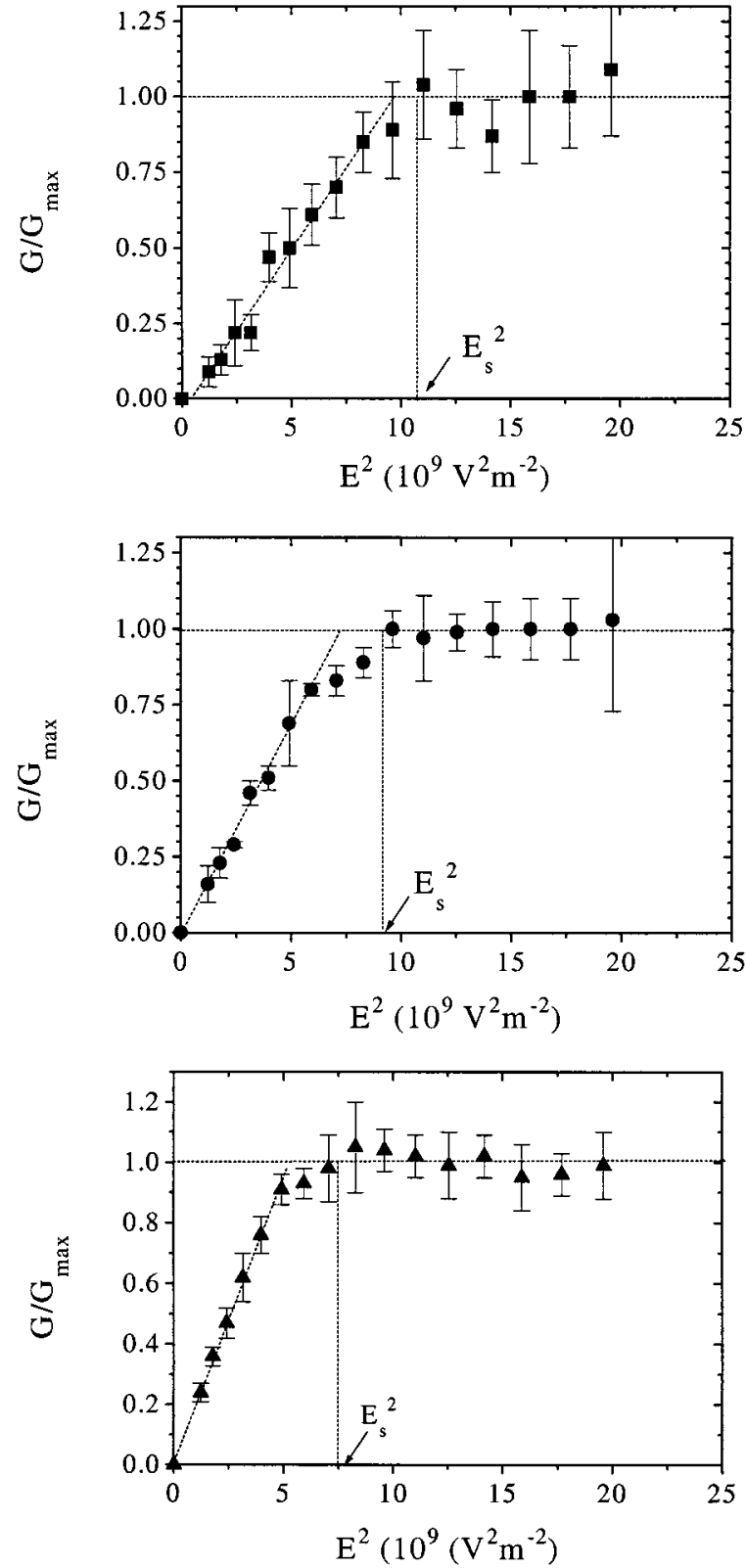

Figure 9. The experimental normalized electro-optic response $\left(G / G_{\max }\right)$ as a function of the electric field strength squared $\left(E^{2}\right)$ for the dispersions

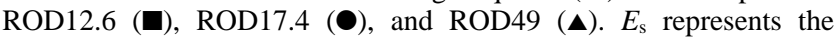
saturation voltage.

$\Delta \alpha=6 \cdot 10^{-30} \mathrm{~F} \cdot \mathrm{m}^{2}$ to $\Delta \alpha=12 \cdot 10^{-30} \mathrm{~F} \cdot \mathrm{m}^{2}$, whereas the electrical field strength at which the steady-state value is reached becomes smaller with increasing particle length. Table 2 summarizes the anisotropy in electric polarizabilities and the saturation electrical field strengths. The $\Delta \alpha$ values are similar to the polarizability of viruses like TMV. ${ }^{1,7}$ The mechanism of alignment of viruses induced by electric fields is ascribed to the double-layer polarization. , $, 14,31$ Presumably, the double layer polarization plays a role in the alignment of gold rods as well. Table 2 includes the results obtained by Zhivkov et a1., ${ }^{23}$ who performed electric birefringence on colloidal gold rods with $L / d$ $=17.2$. The particle characteristics are given in Tables 1 and 2. The polarizability deduced from the change in transverse resonance is roughly in agreement with that measured by Zhivkov et a1.23

We found that the anisotropy in electrical polarizability depends on the square root of the particle length indicated by the line through the origin in Figure 11. More variables such
TABLE 2: Electro-Optical Data: Experimental Values for the Anisotropy in Electric Polarizability $(\Delta \alpha)$ and Saturation Electrical Field Strength $\left(E_{\mathrm{s}}\right)^{a}$

\begin{tabular}{lccccc}
\hline & $\begin{array}{c}\Delta \alpha \\
\left(10^{-30}\right. \\
\left.\mathrm{Fm}^{2}\right)\end{array}$ & $\begin{array}{c}\mathrm{E}_{\mathrm{s}} \\
(\mathrm{V} / \mathrm{mm})\end{array}$ & $\begin{array}{c}\lambda \\
(\mathrm{nm})\end{array}$ & $\begin{array}{c}\omega \\
(\mathrm{kHz})\end{array}$ & technique $^{a}$ \\
\hline ROD12.6 & 6.6 & 110 & 517 & 10 & transverse resonance \\
ROD17.4 & 8.3 & 95 & 517 & 10 & transverse resonance \\
ROD49 & 12 & 87 & 517 & 10 & transverse resonance \\
ROD17.2 & 11.6 & & 650 & 2.5 & birefringence $^{23}$ \\
$c$ & 9.7 & & 650 & 50 & birefringence $^{23}$
\end{tabular}

${ }^{a} \lambda=$ the wavelength at which the measurement is performed. $\omega=$ the frequency of the ac voltage used in the measurement. ${ }^{b}$ The techniques with which $\Delta \alpha$ is determined are indicated. Note: $\Delta \alpha$ calculated from the transverse resonance is overestimated with about $10 \%$ (see Figure 10) because $G_{\max }<G_{\infty}{ }^{c}$ The bottom line refers to the results discussed in ref 23 .

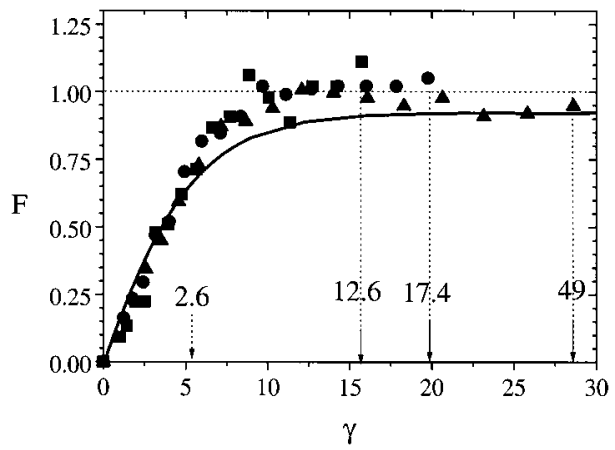

Figure 10. The orientation factor of the gold rods for the dispersions

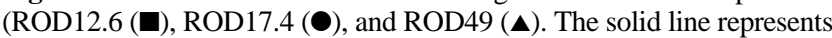
the curve calculated by O'Konski ${ }^{1}$ (see Figure 3 ). The arrows indicate the maximum values for the $\gamma$ in the experiments for various aspect ratios. The numbers on the arrows refers to the aspect ratio of the rods.

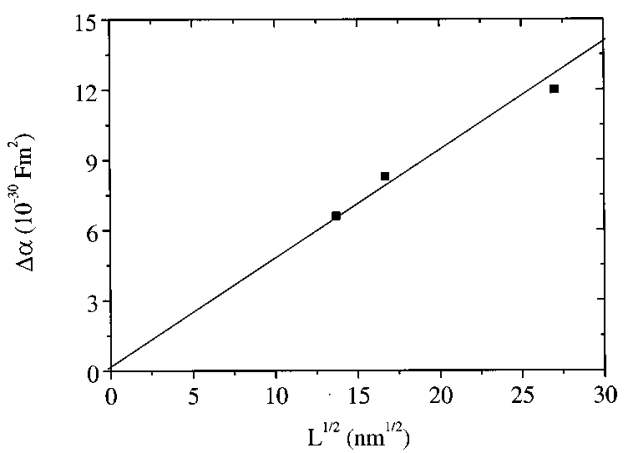

Figure 11. The electric polarizability $(\Delta \alpha)$ plotted against the square root of the length $\left(L^{1 / 2}\right)$.

as the effect of diameter, aspect ratio, and salt concentration have to be investigated in order to understand the physical meaning of the square root dependence. We note here that the salt concentration was not systematically controlled, but indications have been obtained that small changes in salt concentration do not significantly affect the double layer properties: the $\zeta$ potential was constant for all dispersions.

Structure Formation Induced by Enhanced Electric Fields. Although the effect of the duration of the electric field was not examined in detail, we here qualitatively discuss the observations performed by eye on a dispersion with gold rods of $L / d=39$. For $50 \mathrm{~V}$ and a frequency of $100 \mathrm{kHz}$, visually nothing changes on the time scale of seconds. However, if the field was applied for 1-2 min, we observed the development of a structure. This structure was composed of alternating strings of concentrated and less concentrated regions. When the electric field was switched off, the dispersion randomnized and the 
experiment could be repeated. A similar behavior was found for rods with $L / d=32$ and $L / d=10$ stabilized with polyacrylic acid $\left(\right.$ PAA, $M_{\mathrm{w}}=2000$ ) at ac voltages of $10 \mathrm{~V}$ and $1 \mathrm{kHz}$ as well as for $10 \mathrm{~V}$ and $1 \mathrm{MHz}$.

String formation is often observed in electrorheological fluids, which are in general nonaqueous media. ${ }^{30,31}$ The applied electric field induces dipoles in the particles. These induced dipoles give rise to long-range interactions and to lower the free energy; the particles form strings. ${ }^{31,32}$

\section{Conclusions}

Electrical alignment of gold rods dispersed in an aqueous medium is achieved, resulting in a strong electro-optical effect, which is in agreement with theory. It is found that the transverse absorbance band is very sensitive to orientational order. Even in the pseudosaturation regime a small shoulder is present, demonstrating that complete alignment in aqueous systems is difficult to achieve. The magnitude of the shoulder depends on the particle length. The anisotropy in electric polarizability deduced from the transverse resonance varies from $6 \cdot 10^{-30}$ to $12 \cdot 10^{-30} \mathrm{~F} \cdot \mathrm{m}^{2}$ for rods with a length in range of 189 to 730 $\mathrm{nm}$, which is similar to the electric polarizabilities of viruses such as TMV. It appears that the anisotropy in electrical polarizability depends on the square root of the particle length. Apart from the change in the transverse resonance band, the spectral curve shifts to higher absorbance values.

Acknowledgment. The authors thank Dr. H. Gerritsen and Dr. M. van Zandvoort for the opportunity to perform the measurements in their Molecular Biophysics group in Utrecht. Dr. P. Mulvaney and Dr. D. Dunstan both from the University of Melbourne are acknowledged for their assistance with the preliminary experiments. Prof. S. Stoylov and Dr. A. Zhivkov are thanked for their valuable comments on the theory and experiments. Dr. Ir. L. G. J. Fokkink (Philips Research, Eindhoven, PRL), Dr. Ir. M. R. Böhmer (PRL), and Prof. Dr. A. P. Philipse (Van't Hoff laboratory for Physical and Colloid Chemistry, Utrecht University) are acknowledged for stimulating discussions. This work is part of the research program of the Foundation for Fundamental Research on Matter (FOM) with financial support from the Netherlands Organization for Scientific Research (NWO).

\section{References and Notes}

(1) O'Konski, C. T.; Yoshioka, K.; Orttung, W. H. J. Phys. Chem. 1959, 63, 1558.
(2) Asai, H.; Watanabe, N. Biopolymers 1976, 15, 383.

(3) Radeva, T. J. Colloid Interface Sci. 1997, 187, 57.

(4) Peikov, V.; Petkanchin, I.; Stoylov, S. P. J. Disp. Sci. Techn. 1993, 14,499 136.

(5) van de Ven, T. G. M.; Baloch, M. K. J. Colloid Interface Sci. 1990 205.

(6) Fairey, R. C.; Jennings, B. R. J. Colloid Interface Sci. 1982, 85,

(7) Stoylov, S. P. Colloid electro-opticals, theory, techniques, applications; Academic Press: New York, 1991.

(8) Stoylov, S. P. Adv. Collioid Interface Sci. 1971, 3, 45.

(9) Fredericq, E.; Houssier, C. Electric dichroism and electric birefringence; Harrington, W., Peacocke, A. R., Eds.; Oxford University Press: Oxford, 1973.

(10) Mantegazza, F.; Degiorgio, V.; Giardini, M. E.; Price, A. L.; Steytler, D. C.; Robinson, B. H. Langmuir 1998, 14, 1.

(11) Radeva, T.; Petkanchin, I.; Varoqui, R. Langmuir 1993, 9, 170.

(12) Heller, W. Rev. Modern Phys. 1942, 14, 390.

(13) Hoffmann, H.; Krämer, U.; Thurn, H. J. Phys. Chem. 1990, 94, 2027.

(14) Krämer, U.; Hoffmann, H. Macromolecules 1991, 24, 256.

(15) Radeva, T.; Stoimeva, M. Colloid Surf. 1991, 54, 235.

(16) Stoimeva, M.; Radeva, T. J. Colloid Interface Sci. 1991, 141, 433.

(17) Stoimeva, M.; Zhivkov, A. J. Colloid Interface Sci. 1991, 142, 92.

(18) Stoimeva, M.; Radeva, T. J. Colloid Interface Sci. 1995, 169, 329.

(19) van der Zande, B. M. I.; Pagès, L.; Hikmet, R. A. M.; van Blaaderen, A. J. Phys. Chem. B 1999, 103, 5761 (following paper in this issue).

(20) Fornasiero, D.; Grieser, F. Chem. Phys. Lett. 1987, 139, 103.

(21) Thulstrup, E. W.; Michl, J.; Eggers, J. H. J. Phys. Chem. 1970, 74, 22

(22) van der Zande, B. M. I.; Bohmer, M. R. Manuscript in preparation.

(23) Zhivkov, A. M.; van der Zande, B. M. I.; Stoylov, S. P. Manuscript in preparation.

(24) Peterlin, A.; Stuart, H. A. Z. Phys. 1939, 112, 129.

(25) van der Zande, B. M. I.; Böhmer, M. R.; Fokkink, L. G. J.; Schönenberger, C. J. Phys. Chem. B 1997, 101, 852. van der Zande, B. M. I.; Böhmer, M. R.; Fokkink, L. G. J.; Schönenberger, C. Manuscript in preparation.

(26) van der Zande, B. M. I.; Dhont, J. K. G.; Böhmer, M. R.; Philipse, A. P. Manuscript in preparation.

(27) Lide, D. R. CRC Handbook of chemistry and physics, 73th ed.; CRC Press: Boca Raton, FL, 1992-1993.

(28) Frens, G. Nature (London), Phys. Sci. 1973, 241, 20.

(29) Otsubo, Y.; Edamura, K.; Akashi, K. J. Colloid Interface Sci. 1996, $177,250$.

(30) Saito, Y.; Hirata, M.; Tada, H.; Hyodo, M. Appl. Phys. Lett. 1993, $3,1319$.

(31) O'Konski, C. T. J. Phys. Chem. 1960, 64, 605

(32) Sprecher, A. F.; Carlson, J. D.; Conrad, H. Mater. Sci. Eng. 1987, 95, 187. 